\title{
BioéthiqueOnline
}

\section{L'ergothérapeute : un professionnel de la santé? Vraiment? Dans quelle mesure?}

\section{Marie-Josée Drolet}

Volume 6, 2017

URI : https://id.erudit.org/iderudit/1044615ar

DOI : https://doi.org/10.7202/1044615ar

Aller au sommaire du numéro

Éditeur(s)

BioéthiqueOnline

ISSN

1923-2799 (numérique)

Découvrir la revue

Citer cet article

Drolet, M.-J. (2017). L'ergothérapeute : un professionnel de la santé? Vraiment? Dans quelle mesure? BioéthiqueOnline, 6. https://doi.org/10.7202/1044615ar
Résumé de l'article

Comme maints professionnels de la réadaptation, l'ergothérapeute est d'emblée considéré comme un professionnel de la santé. Or cette association entre l'ergothérapie et la santé peut être nuancée, voire contestée. 


\title{
L'ergothérapeute : un professionnel de la santé? Vraiment? Dans quelle mesure?
}

\author{
COMMENTAIRE CRITIQUE / CRITICAL COMMENTARY (RÉVISION PAR LES PAIRS / PEER-REVIEWED) \\ Marie-Josée Drolet $^{1}$
}

Reçu/Received: 6 Mar 2017

Publié/Published: 6 Jul 2017

Éditrice/Editor: Aliya Affdal

Évaluateurs externes/Peer-Reviewers: Cynthia Engels

2017 M-J Drolet, Creative Commons Attribution 4.0 International License

\section{Résumé}

Comme maints professionnels de la réadaptation, l'ergothérapeute est d'emblée considéré comme un professionnel de la santé. Or cette association entre l'ergothérapie et la santé peut être nuancée, voire contestée.

\section{Mots clés}

ergothérapie, valeur, occupation, professionnel, santé, justice

\section{Summary}

As with many rehabilitation professionals, the occupational therapist is immediately considered as a health professional. However, this association between occupational therapy and health may be nuanced or even contested.

\section{Keywords}

occupational therapy, value, occupation, professional, health, justice
Responsabilités des évaluateurs externes

Les évaluations des examinateurs externes sont prises en considération de façon sérieuse par les éditeurs et les auteurs dans la préparation des manuscrits pour publication. Toutefois, être nommé comme examinateur n'indique pas nécessairement l'approbation de ce manuscrit. Les éditeurs de BioéthiqueOnline assument la responsabilité entière de l'acceptation finale et la publication d'un article.
Peer-reviewer responsibilities

Reviewer evaluations are given serious consideration by the editors and authors in the preparation of manuscripts for publication. Nonetheless, being named as a reviewer does not necessarily denote approval of a manuscript; the editors of BioéthiqueOnline take full responsibility for final acceptance and publication of an article. 
Affiliations des auteurs / Author Affiliations

${ }^{1}$ Département d'ergothérapie de I'Université du Québec à Trois-Rivières, Trois-Rivières (Québec), Canada

\section{Correspondance / Correspondence}

Marie-Josée Drolet, marie-josee.drolet@uqtr.ca

\section{Remerciements}

Je remercie chaleureusement tous les ergothérapeutes qui ont participé aux recherches que j'ai réalisées sur les valeurs de l'ergothérapie et celles des ergothérapeutes québécois. Je remercie également l' Université du Québec à Trois-Rivières (UQTR) qui m'a offert des fonds m'ayant permis de réaliser ces recherches. Mes remerciements s'étendent aussi à Anick Sauvageau, professeure au Département d'ergothérapie de I'UQTR, qui a commenté la version préliminaire de ce commentaire. Je remercie enfin l'éditrice et la réviseure de ce commentaire pour leurs judicieuses suggestions qui m'ont permis de bonifier mon texte.

\section{Conflit d'intérêts}

Ce commentaire critique n'est lié à aucun conflit d'intérêts. Mentionnons toutefois que les recherches actuelles de l'auteure sur les enjeux éthiques de la pratique de l'ergothérapie sont financées par le Fonds de recherche du Québec - Société et culture (FRQSC), par I'UQTR ainsi que par la Fondation de I'UQTR. Des recherches antérieures sur les valeurs des ergothérapeutes ont été subventionnées par divers Fonds de recherche de I'UQTR, notamment deux Fonds Institutionnels de Recherche (FIR) et trois Fonds d'Animation à la Recherche (FAR).

\section{Acknowledgements}

I would like to warmly thank all the occupational therapists who have participated in my research about the values of occupational therapy and those of Quebec occupational therapists. I also would like to thank the Universite du Québec à Trois-Rivières (UQTR) for providing me with funds to conduct this research. My thanks also go to Anick Sauvageau, professor in the UQTR Department of Occupational Therapy, for her comments on the preliminary version of this commentary. Finally, I would like to thank the editor and the reviewer for their pertinent suggestions which allowed me to improve this text.

\section{Conflicts of Interest}

This critical commentary is not related to any conflict of interest. However, the author's current research on ethical issues in occupational therapy practice are funded by the Fonds de recherche du Québec - Société et culture (FRQSC), the UQTR and by the UQTR Foundation. Previous research on the values of occupational therapists has been funded by various UQTR Research Funds, including two Fonds Institutionnels de Recherche (FIR) and three Fonds d'Animation à la Recherche (FAR).

\section{Introduction}

L'ergothérapeute ${ }^{1}$ est un professionnel de l'habilitation à l'occupation, c'est-à-dire un professionnel de la réadaptation qui vise à aider les personnes ou les communautés à choisir, organiser et réaliser les activités qu'elles considèrent utiles ou importantes et qui donnent un sens à leur existence [1]. Comme l'indique la Fédération mondiale des ergothérapeutes, le principal but de l'ergothérapeute est d'habiliter les personnes ou les communautés à prendre part aux activités de la vie quotidienne, en soutenant le développement de leurs capacités, en modifiant leurs occupations ou en adaptant les environnements au sein desquels les personnes ou les communautés réalisent leurs activités [2].

Ce texte articule une réflexion critique sur le fait d'associer d'emblée l'ergothérapie à une profession de la santé. Celle-ci découle de recherches que j'ai réalisées sur les valeurs de l'ergothérapie [3] et celles d'ergothérapeutes du Québec [4-8]. La thèse que je défends dans ce commentaire est la suivante: je considère que cette association peut et doit être nuancée, voire contestée pour différentes raisons que je développe ici. La première a trait à l'histoire de la profession (raison historique). La deuxième est liée aux valeurs de la profession (raison axiologique). La troisième est relative à la socialisation professionnelle de l'ergothérapeute dans divers milieux de pratique (raison psychologique), tandis que la dernière raison concerne la mission sociale de l'ergothérapie, voire de l'ergothérapeute (raison sociale).

\section{Brève histoire de l'ergothérapie}

L'ergothérapie tire son origine des sciences médicales (notamment la médecine et les sciences infirmières) et des sciences sociales (spécialement le travail social) [9-10]. Ainsi, depuis ses tout

\footnotetext{
${ }^{1}$ Dans ce commentaire, le masculin est utilisé afin d'alléger le texte, et ce, sans aucune discrimination.
} 
débuts, la profession ergothérapique est en quelque sorte tiraillée entre ses valeurs, modalités et modèles biomédicaux ainsi que ses valeurs, modalités et modèles sociaux. Encore de nos jours, cette double origine, voire cette tension interne à la profession peut être constatée en pratique. Par exemple, on observe habituellement que la pratique de l'ergothérapie en santé physique aura tendance à opter pour des modalités thérapeutiques et modèles conceptuels plus biomédicaux, tandis que la pratique de l'ergothérapie en santé mentale aura plutôt tendance à s'arrimer à des modalités thérapeutiques et modèles conceptuels plus sociaux, voire artistiques. En dépit de ces différences dans le choix des modèles conceptuels et des modalités thérapeutiques, il n'en demeure pas moins que les valeurs au fondement de la profession sont davantage sociales que biomédicales, comme nous le verrons au point suivant. En bref, ce sont les philosophies et les pratiques des établissements qui en viennent petit à petit à socialiser l'ergothérapeute de diverses manières [6], parfois de façon cohérente à ses valeurs sociales, parfois de façon plus ou moins éloignée de celles-ci. Comme l'ergothérapeute fait montre d'une grande capacité d'adaptation [7], il parvient à s'adapter à ces différents environnements sociaux. Cela dit, il peut être appelé à vivre de la détresse éthique lorsqu'il se présente dans son milieu des barrières qui l'empêchent d'agir de manière cohérente avec sa conscience éthique, c'est-à-dire en cohérence avec ses valeurs sociales fondamentales [8]. Les recherches que j'ai menées jusqu'à maintenant sur les valeurs des ergothérapeutes suggèrent que les ergothérapeutes qui travaillent en santé physique semblent être plus propices à la détresse éthique que ceux qui œuvrent en santé mentale [3-8]. Est-ce parce que les us et coutumes des établissements de santé physique, qu'ils soient publics ou privés, s'arriment davantage à des valeurs biomédicales que les établissements de santé mentale? Je ne suis pas en mesure de répondre actuellement à cette question qui émerge des travaux que j'ai réalisés.

\section{Valeurs professionnelles de l'ergothérapie et des ergothérapeutes}

Lorsque l'on s'intéresse aux valeurs de l'ergothérapie [1,3,11-12] et à celles des ergothérapeutes [48,13-15], force est de constater que la valeur qu'est la santé est certes valorisée dans la profession et par les ergothérapeutes, mais dans une moindre mesure que d'autres valeurs reliées à l'occupation humaine (comme la participation occupationnelle, l'engagement occupationnel, la signifiance occupationnelle, l'équilibre occupationnel et la justice occupationnelle). En effet, lorsqu'on interroge des ergothérapeutes sur leurs valeurs professionnelles, et ce, peu importe leur nationalité, la vaste majorité d'entre eux accorde plus d'importance à la participation occupationnelle (c'est-à-dire au fait pour les patients de prendre part aux activités qui sont importantes pour eux et qui donnent un sens à leur existence) qu'elle n'en accorde à la santé [4,13-15]. C'est que la profession ergothérapique est davantage liée à l'autonomie et au bien-être des patients qu'à leur santé proprement dite. Ce faisant, la profession accorde une grande importance aux valeurs des patients ainsi qu'à leurs projets occupationnels et à leurs activités quotidiennes. Cette posture axiologique éloigne l'ergothérapeute d'une profession de la santé pour le rapprocher d'une profession à caractère social, comme nous le verrons au dernier point.

Bien entendu, argueront certains, le fait de participer et de s'engager dans de tels projets et activités contribue en règle générale à la santé des patients. Comme l'affirme d'ailleurs la Fédération mondiale des ergothérapeutes, l'ergothérapeute est un professionnel centré sur le patient qui promeut la santé par l'entremise de l'occupation [2]. Or ce n'est pas toujours le cas [16-19]. De fait, les êtres humains réalisent d'innombrables activités qui nuisent à leur santé ou qui posent un risque pour leur intégrité physique, cognitive ou affective, mais ces activités n'en demeurent pas moins importantes et signifiantes pour eux. Que l'on pense aux personnes qui pratiquent un sport extrême, aux individus qui consomment en grande quantité des boissons ou des aliments peu sains pour la santé, aux personnes qui ont des passions faisant en sorte que leur horaire occupationnel n'est pas équilibré ou encore aux individus qui passent un grand nombre d'heures assis devant un écran, ces illustrations assez fréquentes ne constituent que des exemples. Somme toute, l'ergothérapeute n'est pas là pour juger les choix occupationnels des patients. Dans la mesure où ces choix ne nuisent pas à d'autres êtres humains, il va en général de soi pour l'ergothérapeute de soutenir les projets occupationnels 
des patients, c'est-à-dire d'adopter une approche centrée sur le patient qui l'amène à respecter l'autonomie décisionnelle des patients, à valoriser leur voix et leur vision particulière des choses.

C'est que l'ergothérapeute considère que les occupations humaines sont idiosyncrasiques, qu'elles sont porteuses d'un sens qui est propre aux personnes et aux communautés. Cette posture épistémologique conduit en général l'ergothérapeute à être réfractaire à toute forme de paternalisme, fût-il médical ou éthique. II s'ensuit qu'il aura tendance à valoriser la liberté individuelle et la diversité humaine. Ce faisant, il sera dans bien des cas un défenseur et un promoteur de la justice occupationnelle, c'est-à-dire qu'il travaillera à habiliter les environnements physiques et sociaux ainsi qu'à modifier les occupations afin d'offrir des opportunités occupationnelles équitables aux personnes, par-delà leurs différences, déficiences ou incapacités. Une personne quadriplégique souhaite-t-elle faire du ski alpin ou de la voile? L'ergothérapeute tentera de trouver des adaptations pour soutenir ce patient dans son projet, même si ce faisant cette activité comporte des risques pour sa sécurité. Un patient estime-t-il que la consommation d'une certaine drogue le met dans une disposition créative lui permettant de s'engager à fond dans le projet artistique qui lui tient à cœur? L'ergothérapeute cohérent avec les valeurs de la profession n'aura pas tendance à le moraliser. II voudra certes encadrer les comportements autodestructeurs qui empêchent la réalisation d'occupations, mais il sera en général sensible et ouvert au sens que chacun accorde aux activités qui meublent son quotidien et qui contribuent à actualiser son individualité et son bien-être. C'est que l'ergothérapeute valorise la participation et la signifiance occupationnelles, car il est un occupational therapist ou un ergon (travail, activité ou occupation en grec) thérapeute qui considère que l'être humain est un être occupationnel $[1,3,12]$.

\section{Milieux de pratique des ergothérapeutes}

Traditionnellement, l'ergothérapeute a travaillé dans le système de santé. Cependant, de plus en plus d'ergothérapeutes interviennent dans d'autres milieux que ceux liés à la santé. En effet, de nos jours, les ergothérapeutes travaillent par exemple dans les milieux scolaires et communautaires ainsi que dans les milieux de vie des personnes [20-21]. C'est l'origine de l'ergothérapie combinée au contexte social qui ont fait en sorte que l'ergothérapeute a traditionnellement évolué dans le système de santé. Or de plus en plus d'ergothérapeutes investissent d'autres milieux plus psychosociaux, moins biomédicaux. Ce faisant, ils actualisent leurs idéaux éthiques, leur essence axiologique. Les ergothérapeutes qui œuvrent dans ces milieux cherchent à habiliter les personnes et les communautés à participer aux occupations que ces dernières valorisent ou qui font partie de leur quotidien. C'est que l'ergothérapeute est un professionnel de l'habilitation à l'occupation, disions-nous plus tôt. II valorise l'émancipation des personnes et des communautés par l'entremise de leur participation et de leur engagement occupationnels, lesquels contribuent, estime-t-il, à la justice sociale, à l'équité et à l'inclusion sociale ainsi que dans bien des cas, mais pas tout le temps, à la santé. Bien entendu, l'ergothérapeute valorise la santé et travaille à la santé de patients, mais la santé a en général pour lui un rôle instrumental. C'est parce que la santé permet la réalisation et l'engagement des personnes et des communautés dans des activités porteuses de sens et importantes pour elles que celle-ci a une valeur. Autrement dit, la participation, la signifiance, la justice et l'engagement occupationnels ont pour l'ergothérapeute une valeur intrinsèque, une valeur en eux-mêmes, alors que la santé a en général une valeur extrinsèque, une valeur instrumentale.

\section{Ergothérapeute : un artisan de la justice sociale}

Pour le dire clairement : l'ergothérapeute est d'abord et avant tout un artisan de la justice sociale en tant que justice occupationnelle. Les différents mémoires rédigés par l'Ordre des ergothérapeutes du Québec sont en ce sens emblématiques [22]. Ils rappellent que l'ergothérapeute est un ardent défenseur de la justice sociale, de l'équité et de l'inclusion sociale. De plus en plus, l'ergothérapeute adopte, ou est invité à adopter, un rôle plus politique afin que toute personne, par-delà ses capacités ou incapacités, puisse contribuer à la vie collective et prendre part à sa mesure aux occupations 
qu'elle estime importantes et signifiantes. Ce faisant, l'ergothérapeute réalise ou est encouragé à réaliser des activités de promotion et de défense des droits occupationnels des patients (des activités d'advocacy), et ce, en collaboration et à la demande de ces derniers [23]. L'ergothérapeute est ainsi amené à combattre la discrimination, la stigmatisation, la marginalisation, la domination et toutes autres formes d'injustices sociales ou d'oppression. Lorsqu'il s'engage dans de telles activités, l'ergothérapeute se trouve alors en concordance avec ses valeurs éthiques fondamentales, son essence sociale. Ainsi, bien que l'ergothérapeute contribue dans bien des cas à la santé humaine et qu'il ait traditionnellement travaillé dans le système de santé, cet amalgame entre l'ergothérapie et la santé ne rend pas entièrement justice à l'ontologie axiologique de la profession, c'est-à-dire aux valeurs qui sont constitutives de l'essence de l'ergothérapie, lesquelles sont davantage liées à la justice sociale, à l'équité et à l'inclusion sociale qu'à la santé.

\section{Conclusion}

Pour conclure, c'est à cette découverte que mes recherches sur les valeurs de la profession d'ergothérapeute et celles d'ergothérapeutes québécois m'ont en outre menée. Peut-être que plusieurs ergothérapeutes ont depuis longtemps compris cette réalité - à savoir que la valeur qu'est la santé est certes importante en ergothérapie, mais celle-ci demeure accessoire dans une certaine mesure - mais en ce qui me concerne cette découverte est récente. II m'est apparu important de la partager, car je pense que l'association systématique de l'ergothérapie à une profession de la santé peut empêcher de percevoir ce qui constitue l'essentiel de la profession d'ergothérapeute, soit que l'occupation humaine est centrale en ergothérapie, car c'est elle qui donne un sens à l'existence des êtres humains. Lever le voile sur cette réalité axiologique pourrait peut-être aider les collègues des ergothérapeutes à mieux comprendre la posture éthique des ergothérapeutes ainsi que les décisions que ces derniers prennent et les actions qu'ils posent. Comme cette confusion est susceptible d'éloigner l'ergothérapeute de l'essence de la profession, éclairer cette réalité a aussi le potentiel d'aider certains ergothérapeutes à se rappeler, au besoin, leur origine et leur essence. Car comme le disait Socrate à propos de la maïeutique, apprendre c'est se ressouvenir [24]. Et puisque se ressouvenir permet de bien agir, car nul ne fait le mal volontairement [25], estimait aussi Socrate, il m'est apparu judicieux de partager ma ressouvenance.

\section{Références}

1. Townsend EA, Polatajko HJ. Habiliter à l'occupation. Faire avancer la perspective ergothérapique de la santé, du bien-être et de la justice par l'occupation. 2nd Ed. Ottawa : CAOT Publications ACE; 2013.

2. World Federation of Occupational Therapists. Statement on occupational therapy. 2010.

3. Drolet MJ. The axiological ontology of occupational therapy: A philosophical analysis. Scandinavian Journal of Occupational Therapy. 2014;21(1):2-10.

4. Drolet MJ, Désormeaux-Moreau M. Les valeurs des ergothérapeutes: Résultats quantitatifs d'une étude pilote exploratoire. BioéthiqueOnline. 2014;3(21):1-16.

5. Drolet MJ, Désormeaux-Moreau M. The values of occupational therapists: Perceptions of occupational therapists in Quebec. Scandinavian Journal of Occupational Therapy. 2016;23(4):272-285.

6. Drolet MJ, Sauvageau A. Developing professional values: perceptions of francophone occupational therapists in Quebec, Canada. Scandinavian Journal of Occupational Therapy. 2016;23(4):286-296.

7. Drolet MJ, Maclure J. Les enjeux éthiques de la pratique de l'ergothérapie: perceptions d'ergothérapeutes. Revue Approches inductives. 2016;3(2):166-196.

8. Drolet MJ, Goulet M. Les barrières et facilitateurs à l'actualisation des valeurs professionnelles: perceptions d'ergothérapeutes du Québec. Recueil annuel belge francophone d'ergothérapie. 2017;9:7-42. 
9. Andersen LT, Reed K. The History of Occupational Therapy: The First Century. Thorofare: SLACK Incorporated; 2016.

10. Friedland J. Restoring the spirit. The Beginnings of Occupational Therapy in Canada, 1890-1930. Montreal \& Kingston, London, Ithaca: McGill-Queen's University Press; 2011.

11. West WL. A reaffirmed philosophy and practice of occupational therapy for the 1980s. American Journal of Occupational Therapy.1984;38:15-23.

12. Yerxa EJ. Audacious values: The energy source for occupational therapy practice. In Kielhofner G, ed. Health Through Occupation: Theory and Practice in Occupational Therapy. Philadelphia: F.A. Davis; 1983, 149-162.

13. Aguilar A, Stupans I, Scutter S. King, S. Exploring professionalism: The professional values of Australian occupational therapist. Australian Occupational Therapy Journal. 2012;59:209-217.

14. Aguilar A, Stupans I, Scutter S. King, S. Towards a definition of professionalism in Australian occupational therapy: Using the Delphi technique to obtain consensus on essential values and behaviours. Australian Occupational Therapy Journal. 2013;60:206-216.

15. Fondiller ED, Rosage LJ, Neuhaus BE. Values influencing clinical reasoning in occupational therapy: An exploratory study. Occupational Therapy Journal Resource. 1990;10:41-55.

16. Fischer TM, Stewart KE, Davis JA. Occupation and health, reconsidered. Canadian Journal of Occupational Therapy. 2014;81(3):140-143.

17. Chang E. Drug use as an occupation: Reflecting on Insite, Vancouver's supervised injection site. Occupational Therapy Now. 2008;10(3):21-23.

18. Elliot M. Figured world of eating disorders: Occupations of illness. Canadian Journal of Occupational Therapy. 2012;79:15-22.

19. Stewart KE, Fischer TM, Hirji R, Davis JA. Toward the reconceptualization of the relationship between occupation and health and well-being. Canadian Journal of Occupational Therapy. 2016;83(4):249-259.

20. Ordre des ergothérapeutes du Québec (OEQ). Mémoire présenté dans le cadre de la consultation publique du ministre de l'Éducation, du Loisir et du Sport sur la réussite éducative. Montréal: OEQ; 2016.

21. Hébert $M$, Maheux $B$, Potvin $L$. Théories qui émergent du quotidien de la pratique communautaire de l'ergothérapie. Canadian Journal of Occupational Therapy. 2002;69:31-39.

22. Ordre des ergothérapeutes du Québec (OEQ). Mémoires. In Publications. Montréal: OEQ. Repéré à http://www.oeq.org/publications/documents-professionnels.fr.html

23. Drolet MJ, Lalancette M, Caty MË. ABC de l'argumentation pour les professionnels de la santé et toute personne qui souhaite convaincre. Québec: Presses de l'Université du Québec; 2015.

24. Platon. Théètète. Paris : Garnier-Flammarion; 2016.

25. Platon. Gorgias. Paris : Garnier-Flammarion; 2010. 